\title{
Inhibition of Mus8I by siRNA enhances sensitivity to 5 -FU in breast carcinoma cell lines
}

This article was published in the following Dove Press journal:

OncoTargets and Therapy

21 October 2014

Number of times this article has been viewed

\author{
Ying Qian ${ }^{1,2}$ \\ Yanning Liu $^{3}$ \\ Qiuyue Yan $^{1,2}$ \\ Juan $L v^{2}$ \\ Xiaoyan $\mathrm{Ni}^{2}$ \\ Yunlu Wu ${ }^{1,2}$ \\ Xuejun Dong ${ }^{1,2}$ \\ 'The Key Laboratory of Laboratory \\ Medicine, Ministry of Education of \\ China; School of Laboratory Medicine \\ and Life Science, Wenzhou Medical \\ University, Wenzhou, Zhejiang, \\ People's Republic of China; ${ }^{2}$ Shaoxing \\ People's Hospital, Shaoxing Hospital \\ of Zhejiang University, Shaoxing, \\ Zhejiang, People's Republic of China; \\ ${ }^{3}$ State Key Laboratory for Diagnosis \\ and Treatment of Infectious Diseases, \\ The First Affiliated Hospital, School \\ of Medicine, Zhejiang University; \\ Collaborative Innovation Center for \\ Diagnosis and Treatment of Infectious \\ Diseases, Hangzhou, Zhejiang, People's \\ Republic of China
}

Correspondence: Xuejun Dong Shaoxing People's Hospital,Shaoxing Hospital of Zhejiang University, 568 Zhongxing North Road, Shaoxing, Zhejiang, 3/2000, People's Republic of China Tel/Fax +86 57588228576 Email dxj9666@।63.com
Purpose: One of the most challenging aspects of breast carcinoma chemotherapy is the rapid acquirement of drug resistance. Improving the sensitivity to chemotherapeutic drugs is very important for successful treatment. Mus 81 plays an important role in maintaining the stability of the genome and DNA repair. However, recent studies suggested that Mus81 expression levels correlate well with resistance to DNA-damaging drugs. The present study aimed to investigate the role of Mus81 on the chemosensitivity of breast carcinoma cells in response to 5-fluorouracil (5-FU), a chemotherapeutic drug that is widely used for treatment of breast malignancies.

Methods: The expression of Mus 81 in MCF-7 and T47D cells was suppressed by small interfering RNA (siRNA). mRNA and protein levels of Mus 81 were analyzed by quantitative real-time polymerase chain reaction and Western blot. Cell viability and colony survival were determined by Cell Counting Kit- 8 and plate colony formation assay, respectively. Cell cycle and apoptosis were detected by flow cytometry.

Results: 5-FU inhibited the cell viability of MCF-7 and T47D cells in a concentration-dependent manner. We found that the Mus81-silenced MCF-7 and T47D cells exhibited decreased cell viability and clonogenic survival, but increased G2 accumulation, in response to 5-FU. In addition, Mus81 deficiency resulted in increased apoptosis and p53 expression in MCF-7 after 5-FU treatment. However, Mus81 deficiency did not affect the apoptosis of T47D cells with 5-FU.

Conclusion: Taken together, our data suggest that Mus 81 inhibition significantly increased the chemosensitivity of MCF-7 and T47D cells in response to 5-FU. Thus, Mus81 siRNA is potentially a useful adjuvant strategy for breast cancer chemotherapy.

Keywords: Mus81, siRNA, 5-FU, breast carcinoma, chemosensitivity, p53

\section{Introduction}

Breast cancer is one of the most common malignant tumors among women all over the world. ${ }^{1,2}$ Combination chemotherapy is a conventional choice for breast cancer after surgery in the clinic. Currently, 5-fluorouracil (5-FU) plus cyclophosphamide and doxorubicin or epirubicin is widely used to treat breast carcinoma. However, some studies have found that breast cancers show different degrees of primary or acquired resistance to 5-FU, 3,4 and high doses of drugs will result in several adverse side effects to healthy cells. Therefore, improving the chemotherapy sensitivity is important for optimized treatment.

The methyl methanesulfonate and ultraviolet sensitive 81 independent gene (MMS and UV sensitive number 81 , Mus81) is widely conserved among eukaryotes. ${ }^{5-8}$ Mus81 protein is a kind of endonuclease that can remove damaged or aberrant DNA fragments to ensure normal DNA replication. ${ }^{9}$ Mus81-deficient embryonic stem cells and mice 
were found to be hypersensitive to mitomycin C (MMC): the survival rate of Mus81 $1^{+/}$and Mus81 ${ }^{-/}$genotypes of embryonic stem cells and mice were significantly lower than the wild type in response to the same dose of MMC. ${ }^{10}$ Disruption of Mus81 gene would increase the sensitivity to MMC and cisplatin, and this sensitivity could be downregulated to normal after expressing Mus81 again. ${ }^{11}$ In addition, the clonogenic survival of Mus81 ${ }^{-/-}$fibroblast cells was decreased by $\mathrm{Cr}$ [VI] (hexavalent chromium) exposure in a dose-dependent manner compared to wild-type controls. ${ }^{12}$ Other studies reported that the expressions of Mus81 in different tumor cells correlated well with their sensitivity to cisplatin; also, Mus81 expression was increased in 5-FU-resistant pancreatic cancer cells. ${ }^{13,14}$ Therefore, a targeting agent that is specific to Mus81 is potentially a promising method for chemosensitivity improvement, especially in Mus81-positive breast carcinoma.

The present study aimed to examine the effect of Mus81 on the chemosensitivity to 5-FU of MCF-7 and T47D cells.

\section{Materials and methods Cell cultures}

The human breast carcinoma cell lines MCF-7 and T47D cells were obtained from the Shanghai Cell Bank of Chinese Academy of Sciences (Shanghai, People's Republic of China). MCF-7 cells were cultured in minimum essential medium ([MEM] Hyclone, MA, USA). T47D cells were cultured in Dulbecco's Modified Eagle's Medium ([DMEM] Hyclone). Both MEM and DMEM were supplemented with 10\% fetal bovine serum (Thermo Fisher Scientific, Waltham, MA, USA.), penicillin (100 U/mL), and streptomycin $(100 \mathrm{mg} / \mathrm{mL})$. Cells were cultured at $37^{\circ} \mathrm{C}$ in a $5 \% \mathrm{CO}_{2}$ atmosphere.

\section{siRNA transfection}

When the cells had grown to $30 \%-80 \%$ confluency, the medium was changed to serum-free and antibiotics-free medium. Mus81 expression was knocked down by transfection with siRNA (Genepharma, Shanghai, People's Republic of China) directed against protein of interest at the final concentration of $100 \mathrm{nM}$. An siRNA duplex that shared no homologous sequences with the target gene was used as a negative control. Transfection was performed using Lipofectamine $^{\circledR} 2000$ (Thermo Fisher Scientific, Waltham, MA, USA) according to the manufacturer's instructions. The efficiency of transfection was detected using inverted fluorescence microscope and flow cytometric assay. The efficiency of inhibition was determined by quantitative real-time (RT) polymerase chain reaction (PCR) and Western blot analysis.
The first Mus81 siRNA ( siMus81) sequence was 5'-CUGCUGAGCACCAUUAAGUTT-3' and 5'-ACUUAAUGGUGCUCAGCAGTT-3'. The second siMus81 sequence is 5'-ACGCGCUUCGUAUUUCA GATT-3' and 5'-UCUGAAAUACGAAGCGC GUTT-3'. The third siMus81 sequence is 5'-GCAGGAGCCAU CAAGAAUATT-3' and 5'-UAUUCUUGAUGG CUCCUGCTT-5'. The control siRNA sequence is 5'-UUCUCCGAACGUGUCACGUTT-3' and 5'-ACGUGACACGUUCGGAGAATT-3'.

\section{Quantitative RT-PCR}

Cells were seeded in a six-well plate at a density of $5 \times 10^{5}$ cells/well in medium containing $10 \%$ fetal bovine serum at $37^{\circ} \mathrm{C}, 5 \% \mathrm{CO}_{2}$. After transfection with Mus 81 siRNAs (siMus81-1, siMus81-2, siMus81-3), control siRNA (siCtrl) for 24 hours, total RNA was extracted. RNA was isolated from the cells using TRIzol ${ }^{\circledR}$ (Thermo Fisher Scientific) and reverse transcribed using the first-strand cDNA synthesis kit (Biomiga, San Diego, USA) according to the manufacturer's protocol. Quantitative RT-PCR was performed with the Lightcycler 480 PCR apparatus (Hoffman-La Roche Ltd, Basel, Switzerland). PCR primers were used as follows: Mus81, forward nucleotide, 5'-TGTGGACATTGGCGAGAC-3', reverse nucleotide, 5'-GCTGAGGTTGTGGACGGA-3'; and $\beta$-actin, forward nucleotide, 5' - ACCCACACTGTGCCCATCTAC-3', reverse nucleotide, 5'-TCGGTGAGGATCTTCATGAGGTA-3'. The abundance of the Mus81 transcript was expressed relative to the control of $\beta$-actin. The experiments were performed independently three times.

\section{Western blot}

Cells were harvested and rinsed with phosphate buffered saline. Cells were lysed for total protein extraction using RIPA lysis buffer (Beyotime, Jiangsu, Nantong, People's Republic of China). The protein concentration was determined by the Bicinchoninic Acid assay (Beyotime, Nantong, People's Republic of China). Equal amounts of proteins were separated using 10\% gel electrophoresis. Then, the proteins were transferred to PVDF membranes (Whatman, Maidstone, Kent, UK), which were blocked in 5\% bovine serum albumin. PVDF membranes were incubated with primary antibodies against Mus81 (1:1,000; Abcam, Cambridge, UK), p53 (1:1,000; Abcam), and $\beta$-actin (1:5,000; Abcam) overnight at $4{ }^{\circ} \mathrm{C}$. After incubations with horseradish peroxidase-conjugated secondary antibodies (1:10,000; Abcam) for 1 hour at room temperature, the blots were developed using the chemiluminescence detection kit ECL-Plus (Thermo Fisher Scientific, New York, USA) according to the manufacturer's instructions. 
Then, blots were exposed to a radiographic film. $\beta$-Actin expression was used as a control.

\section{Cell viability assays}

Cells were seeded $\left(5 \times 10^{3} /\right.$ well $)$ in 96 -well plates and then transfected with siMus 81 for 24 hours. MCF-7 cells were further assembled with 5-FU (Bioer Technology, Hangzhou, People's Republic of China) at concentrations ranging from $0.625 \mu \mathrm{g} / \mathrm{mL}$ up to $10 \mu \mathrm{g} / \mathrm{mL}$ for 48 hours. T47D cells were further incubated with $5-\mathrm{FU}$ at concentrations ranging from $2.5 \mu \mathrm{g} / \mathrm{mL}$ up to $40 \mu \mathrm{g} / \mathrm{mL}$ for 48 hours. Finally, $10 \mu \mathrm{L}$ tetrazolium salt WST- 8 (Cell Counting Kit-8 [CCK-8]; Keygen, Nanjing, People's Republic of China) was added to each well (final volume ratio as $10 \%)$. Optical density was measured at a wavelength of 450 $\mathrm{nm}$ (OD450). Cell viability was calculated as follows:

Viability of cells $=$ Drug-given group OD450 /Control group OD450 $\times 100 \%$.

\section{Plate colony formation assay}

Cells were transfected with siMus81 for 24 hours and were seeded onto six well-plates at a density of 1000 cells per well. Then, MCF-7 cells were treated with $2.5 \mu \mathrm{g} / \mathrm{mL}$ 5 -FU and T47D cells were treated with $25 \mu \mathrm{g} / \mathrm{mL} 5-\mathrm{FU}$ for 4 hours and allowed to grow for 7 days until visible clones appeared. Cell colony formation was assessed using Giemsa solution. The colony containing more than 50 cells was counted and the number of colonies was calculated. The colony formation rate was calculated using the following equation:

Colony formation rate $=($ Number of colonies/Number of seeded cells) $\times 100 \%$.

\section{Apoptosis assay}

Cells were transfected with siMus 81 for 24 hours, seeded onto sixwell plates at a density of $4 \times 10^{5}$ cells per well. 5 -FU $(2.5 \mu \mathrm{g} / \mathrm{mL})$ was added to MCF-7 cells and $25 \mu \mathrm{g} / \mathrm{mL} 5$-FU were added and the cells left for 48 hours. Cells from each group were harvested and diluted with phosphate buffered saline twice. Then, $5 \mu \mathrm{L}$ of fluorescein isothiocyanate and $5 \mu \mathrm{L}$ of propidium iodide (PI) (Annexin V-FITC Kit; Keygen) were added to $500 \mu \mathrm{L}$ of cells. After incubation in the dark for 5-15 minutes at room temperature, flow cytometry was carried out. The experiments were performed independently three times.

\section{Cell cycle}

After transfection and drug treatment, cells were harvested and fixed in $70 \%$ alcohol overnight at $4{ }^{\circ} \mathrm{C}$, incubated with
$100 \mu \mathrm{L}$ trypsin for 30 minutes at $37^{\circ} \mathrm{C}$, and incubated with $400 \mu \mathrm{L}$ PI for 30 minutes in the dark. In the end, cells were analyzed by flow cytometry.

\section{Statistical analysis}

All data were expressed as mean \pm standard deviation, and SPSS 17.0 software was used for statistical analyses. Oneway analysis of variance (ANOVA) and Student's $t$-test were used to analyze the significance between groups. $P<0.05$ was considered statistically significant.

\section{Results}

siMus8I suppresses mRNA and protein expression of Mus8 I

MCF-7 and T47D cells were transfected with siMus81. The FAM fluorescence could be detected in successfully transfected cells (Figure 1A). The transfection efficiency of MCF-7 and T47D cells, which was further confirmed by flow cytometric assay, was $82.47 \%$ and $78.18 \%$, respectively (Figure 1A). Twenty-four hours after transfection, the inhibition efficiency of siMus 81 was measured by quantitative RT-PCR. Compared with the siCtrl group, Mus81 mRNA expression levels of MCF-7 cells in three siMus 81 groups were markedly reduced, to $39.15 \% \pm 1.93 \%, 30.79 \% \pm 2.01 \%$, and $21.48 \% \pm 2.74 \%$, respectively. Western blot analysis also showed that Mus 81 protein expression levels of MCF-7 were significantly decreased after transfection with siMus 81 for 24, 48, and 72 hours (Figure 1B and C). These results showed that siMus 81 could effectively reduce the mRNA and protein levels of Mus81 in MCF-7 cells, and the third sequence of siMus81was the most effective one. As a result, we chose siMus81-3 for subsequent experiments. Mus81 mRNA expression levels of T47D cells in siMus 81 group were reduced to $33.61 \% \pm 2.85 \%$, compared with the siCtrl group. The amount of Mus81 protein also significantly decreased 24, 48, and 72 hours after transfection with siMus81-3 (Figure 1D). The Mus81 mRNA and protein expression levels of both MCF-7 and T47D cells showed significant reduction after siMus 81 transfection.

\section{Inhibition of Mus8 I sensitizes breast cancer cells to 5-FU}

\section{Cell viability assay}

The cell viability of breast cancer cells was examined by CCK-8 analysis. The OD450 values of MCF-7 cells in the siCtrl and siMus 81 groups were $1.39 \pm 0.30$ and $1.31 \pm 0.26$, respectively; the difference between the two groups was 
A

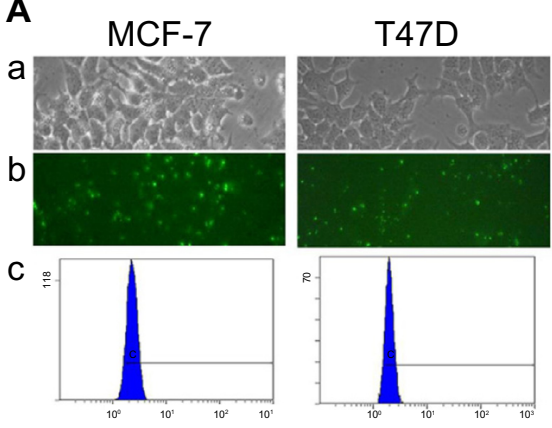

C

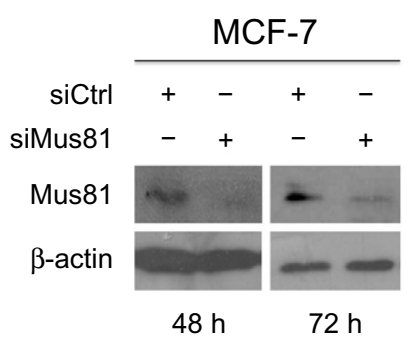

B

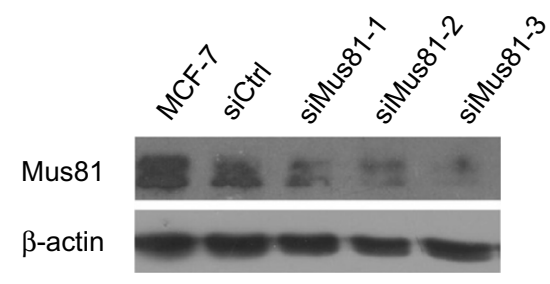

D

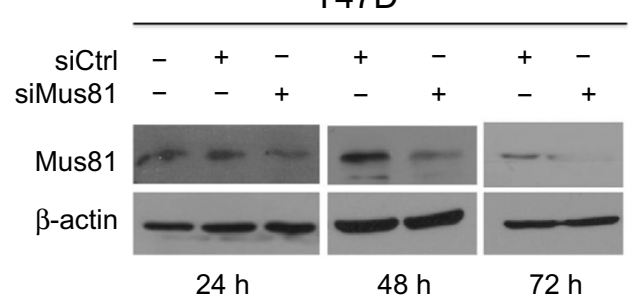

Figure I Inhibition of Mus8I by siRNA.

Notes: (A) MCF-7 and T47D cells were treated with FAM-siRNA. Bright-field images are shown in (a), the corresponding fluorescence images are shown in (b), and flow cytometric analysis images are shown in (c). (B) Western blot analysis of Mus8I protein in MCF-7 cells after 24 hours of siRNA transfection. siMus8I-3 was the most effective siRNA and was chosen for subsequent study. (C) Western blot analysis of Mus8I protein in MCF-7 cells after 48 and 72 hours of siMus8I-3 transfection. (D) Western blot analysis of Mus8I protein in T47D cells after 24, 48, and 72 hours of siMus8I-3 transfection. $\beta$-Actin was used as loading control.

Abbreviations: siMus8I, Mus8I siRNA; siRNA, small interfering RNA; siCtrl, control siRNA; FAM, carboxyl fluorescein.

not statistically significant $(P>0.05)$. Also, the OD450 values of T47D cells in the siCtrl and siMus81 groups were $1.12 \pm 0.34$ and $1.16 \pm 0.31$, respectively; this difference was also not statistically significant $(P>0.05)$. 5-FU could decrease the cell viabilities of MCF-7 and T47D cells in a dose-dependent manner (Figure 2). At the same time, we examined the sensitivity of MCF-7 and T47D cells in the siMus 81 and siCtrl groups to 5-FU. The cell viability in the siMus81 groups was decreased compared to the siCtrl groups in response to 5-FU in both MCF-7 and T47D cells (Figure 2).

\section{Colony formation assay}

We next examined the ability of Mus81-suppressed cells to form colonies by exposure to 5-FU. The clone formation rate of MCF-7 cells in the siMus81 group was significantly lower than in the siCtrl group $(39.97 \% \pm 9.30 \%$ versus $63.23 \% \pm 9.21 \%$, $P<0.05$ ) after 5-FU treatment (Figure $3 \mathrm{~A}$ and $\mathrm{B}$ ). The clone formation rates of T47D cells in the siMus 81 group and siCtrl group were $37.26 \% \pm 7.32 \%$ and $66.33 \% \pm 10.26 \%$, respectively. siMus 81 significantly inhibited colony formation of T47D cells compared with the siCtrl group $(P<0.05)$ (Figure 3C and D).

These data show that Mus81 inhibition resulted in an increased sensitivity of both MCF7 and T47D cells to 5-FU.

\section{Inhibition of Mus8 I affects cell cycle of breast cancer cells with 5-FU}

siMus 81 and siCtrl did not affect the cell cycle of MCF-7 and T47D cells without 5-FU. The G2 proportions of MCF-7 cells with 5-FU in the siCtrl group and in the siMus 81 group were $23.63 \% \pm 1.52 \%$ and $41.81 \% \pm 1.30 \%$, respectively. G2 proportions increased significantly in the siMus 81 group compared with the siCtrl group after 5-FU treatment $(P<0.05)$ (Figure 4A and B). Inhibition of Mus81 also affected cell cycle of T47D cells, and siMus81 combined with 5-FU caused G2 phase accumulation $(P<0.05 ; 30.44 \% \pm 1.55 \%$ for siMus 81 group and $18.31 \% \pm 2.10 \%$ for siCtrl group) (Figure 4C and D).

\section{Inhibition of Mus8I increases apoptosis of MCF-7 cells with 5-FU}

To explore whether chemosensitivity induced by siMus 81 is due to the increase of cell apoptosis, we detected the apoptotic rate in MCF-7 and T47D cells after exposure to 5-FU. Forty-eight hours after drug treatment, flow cytometry (FC) analysis for annexin V/PI revealed a marked increase of apoptotic MCF-7 cells in the siMus81 group compared to the siCtrl group $(41.92 \% \pm 4.82 \%$ versus $27.27 \% \pm 3.98 \%, P<0.05$ ) (Figure $5 \mathrm{~A}$ and $\mathrm{B}$ ). However, there was no difference in the apoptotic rates of T47D cells 
A

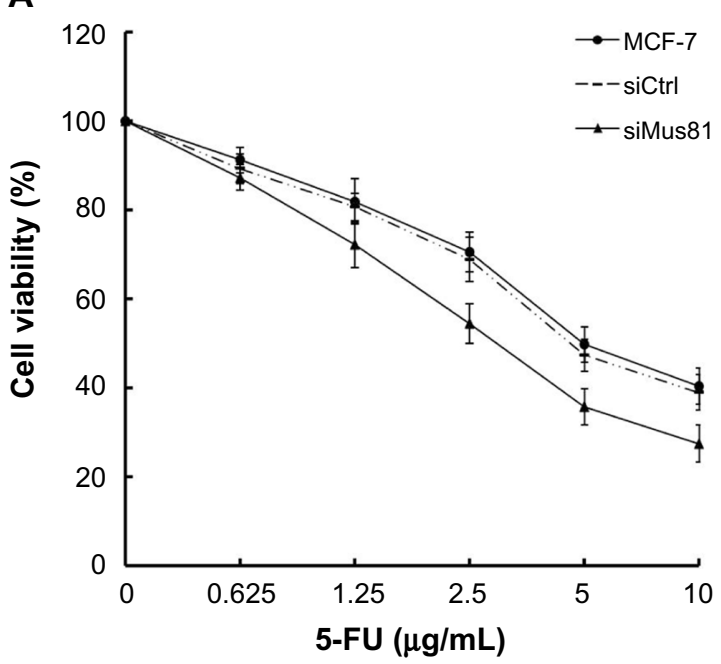

B

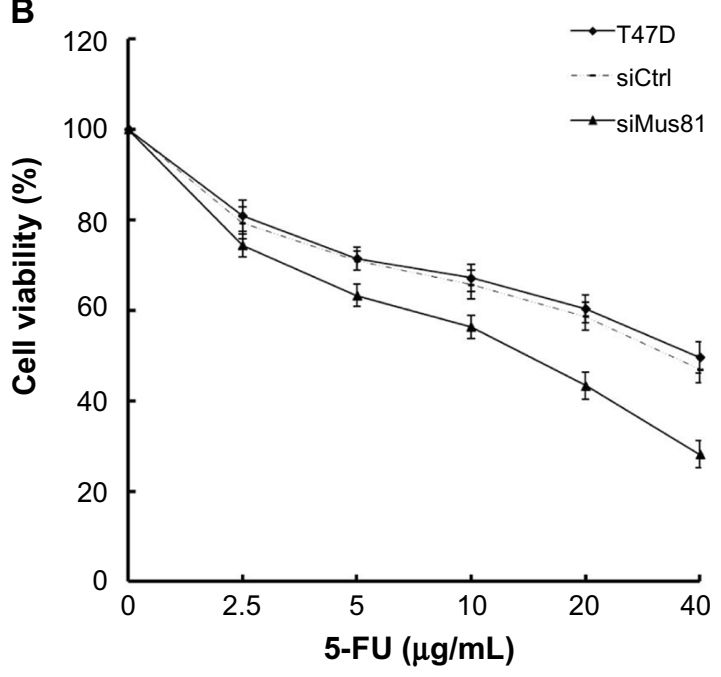

Figure 2 Cell viability assay.

Notes: (A) The cell viability of MCF-7 cells was determined by CCK-8 assay after exposure to 5-FU. Twenty-four hours after siRNA transfection, cells were exposed to 5-FU for 48 hours, and then cell viability was determined by CCK-8 assay. The results were normalized against blank, siCtrl and siMus8I group without 5-FU treatment, respectively (mean $\pm S D, n=3$ ). (B) The cell viability of T47D cells was determined by CCK-8 assay after exposure to 5-FU. Twenty-four hours after siRNA transfection, cells were exposed to 5 -FU for 48 hours, and then cell viability was determined by CCK-8 assay. The results were normalized against blank, siCtrl and siMus $8 \mathrm{I}$ group without 5 -FU treatment, respectively (mean $\pm S D, n=3$ ).

Abbreviations: 5-FU, 5-fluorouracil; CCK-8, Cell Counting Kit-8; siCtrl, control siRNA; siMus8I, Mus8I siRNA; SD, standard deviation; siRNA, small interfering RNA.

between the siMus 81 group and the siCtrl group with 5-FU treatment $(43.57 \% \pm 6.34 \%$ versus $39.87 \% \pm 9.17 \%, P>0.05)$ (Figure 5C and D).

\section{siMus8I-transfected MCF-7 cells express elevated levels of $\mathrm{p} 53$ in response to 5 -FU}

p53 induces cell apoptosis and plays an important role in the response to DNA-damaging agents. Because Mus81

A

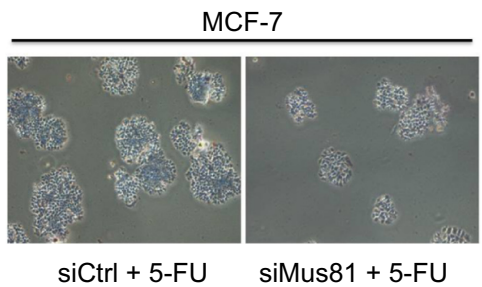

B

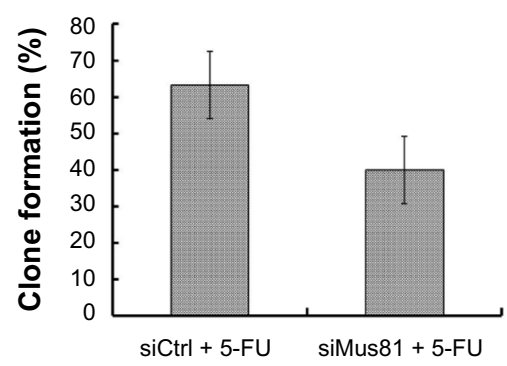

inhibition combined with 5-FU caused increased apoptosis, we examined the p53 expression of MCF-7 in response to 5-FU-induced DNA damage. Similarly to the siCtrl group, the siMus81 group did not show elevated basal levels of p53 expression. However, Western blot analysis indicated that the $\mathrm{p} 53$ expression level of MCF-7 cells increased more in the siMus81 group than in the siCtrl group with 5-FU (Figure 6).

C

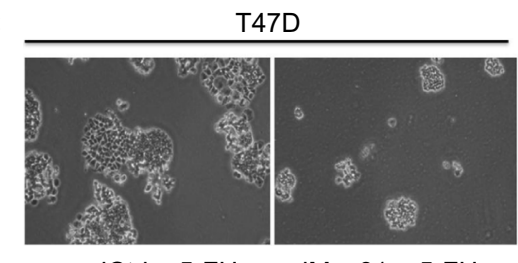

siCtrl + 5-FU

siMus81 + 5-FU

D

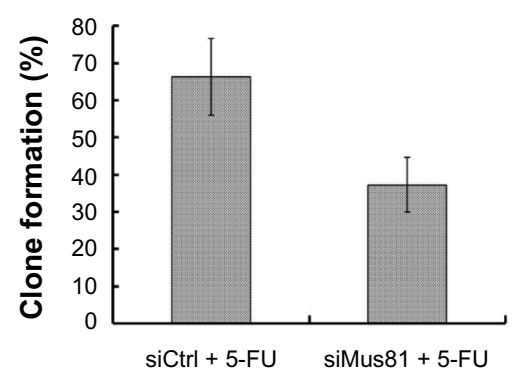

Figure 3 Colony formation assay.

Notes: (A and C) colony forming assay of McF-7 and T47D cells. (B and D) clone formation rate of each group. Colonies were counted on day 7, and analysis was performed (mean $\pm S D, n=3$ ).

Abbreviations: 5-FU, 5-fluorouracil; siCtrl, control siRNA; siMus8I, Mus8I siRNA; SD, standard deviation; siRNA, small interfering RNA. 

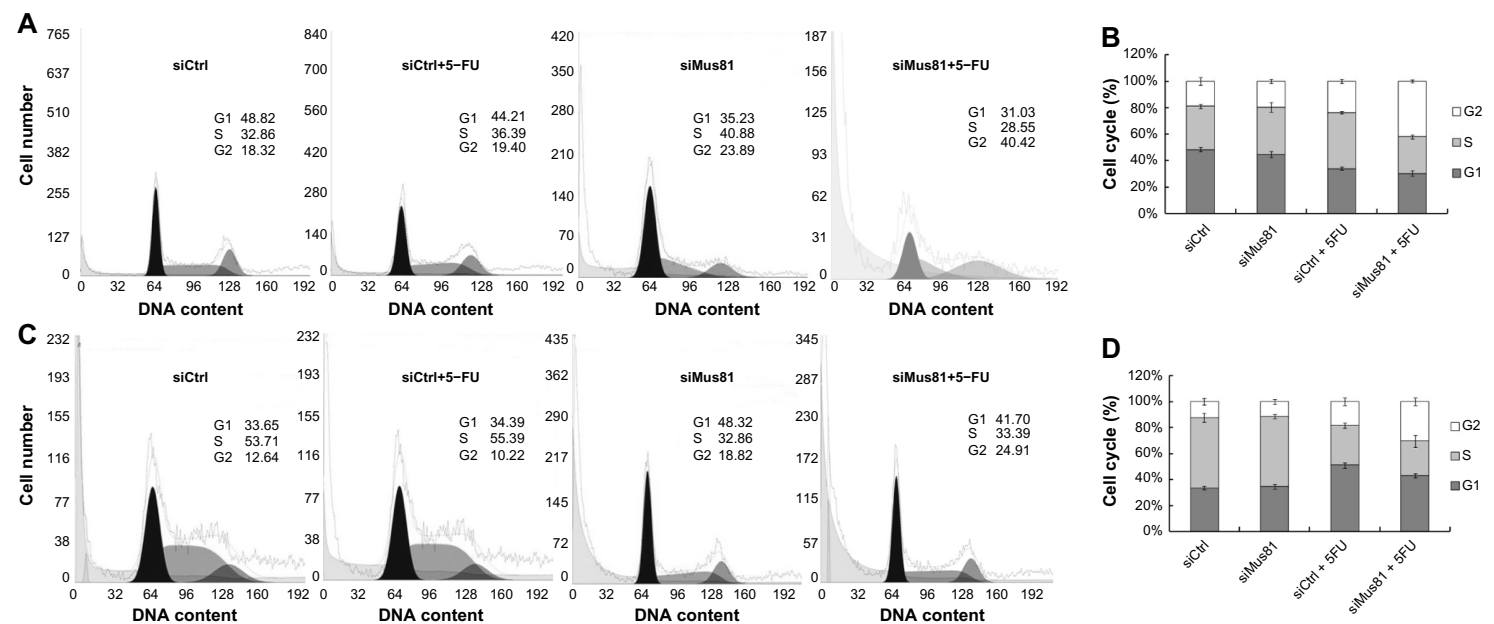

Figure 4 Inhibition of Mus8I affects cell cycle of breast cancer cells with 5-FU.

Notes: Twenty-four hours after transfection, cells were exposed to 5-FU for 48 hours. Cell cycle of MCF-7 cells was analyzed by flow cytometric assay (A), and data are presented as mean \pm SD of three independent experiments (B). Cell cycle of T47D cells was analyzed by flow cytometric assay (C), and data are presented as mean \pm SD of three independent experiments (D). Proportion of G2 phase was increased significantly in the siMus8I group compared with the siCtrl group in both MCF-7 and T47D cells with 5 -FU treatment.

Abbreviations: 5-FU, 5-fluorouracil; siCtrl, control siRNA; siMus8I, Mus8I siRNA; SD, standard deviation; siRNA, small interfering RNA.

\section{Discussion}

In this study, we found that the Mus81-silenced breast cancer MCF-7 and T47D cells exhibited decreased cell viability, clonogenic survival, and G2 accumulation in response to 5-FU. Inhibition of Mus81 only increased apoptosis of MCF-7 cells, not T47D cells treated with 5-FU. We investigated for the first time a mechanism through which the suppression of Mus81 might contribute to p53-mediated chemosensitivity improvement.
Mus81 is a DNA repair gene found in recent years. ${ }^{15}$ Mus81 has been shown to participate in a repair pathway by generation of double-strand break intermediates. Mus81-Eme1/ Mms 4 complex, by pro-cessing stalled replication forks, free 3 '-flap structures, Holliday junctions, and so on, ${ }^{9,16}$ is vital for DNA damage repair and maintenance of chromosomal stability. ${ }^{17}$ Chromosome instability and DNA damage are the main characteristics of cancer cells. ${ }^{18}$ Mammalian cells
A

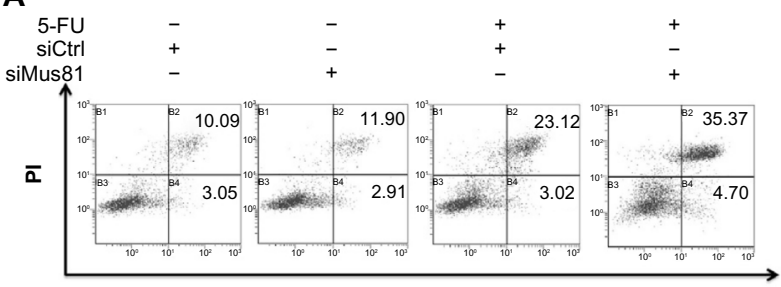

Annexin V

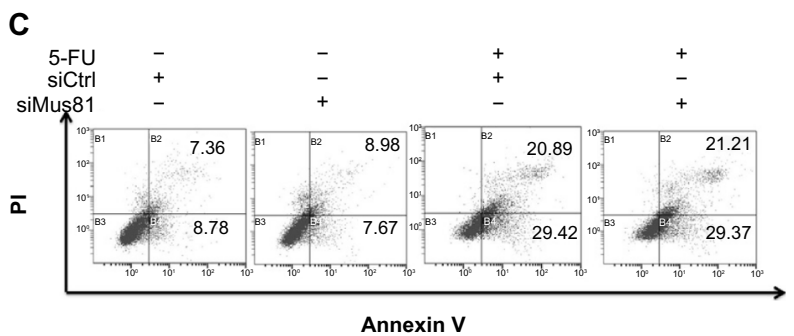

B

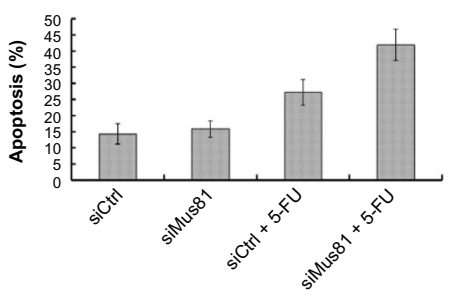

D

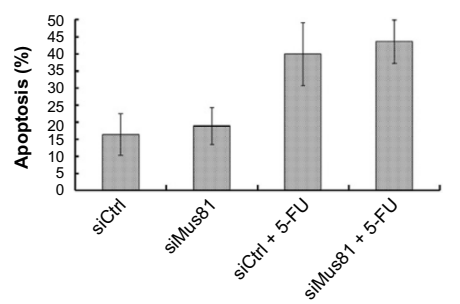

Figure 5 Inhibition of Mus8I affects apoptosis of MCF-7 cells with 5-FU.

Notes: (A) Apoptosis of MCF-7 cells analysis was with combination FITC and annexin V and PI assay. (B) The apoptosis of MCF-7 cells increased in the siMus8I group in response to 5 -FU (mean $\pm \mathrm{SD}, \mathrm{n}=3$ ). (C) Apoptosis of T47D cells was analyzed by FITC-annexin V/PI assay. (D) The apoptosis of T47D cells did not increase in the siMus8I group in response to $5-\mathrm{FU}$ (mean $\pm \mathrm{SD}, \mathrm{n}=3$ ).

Abbreviations: 5-FU, 5-fluorouracil; FITC, fluorescein isothiocyanate; PI, propidium iodide; siCtrl, control siRNA; siMus8I, Mus8I siRNA; SD, standard deviation; siRNA, small interfering RNA. 


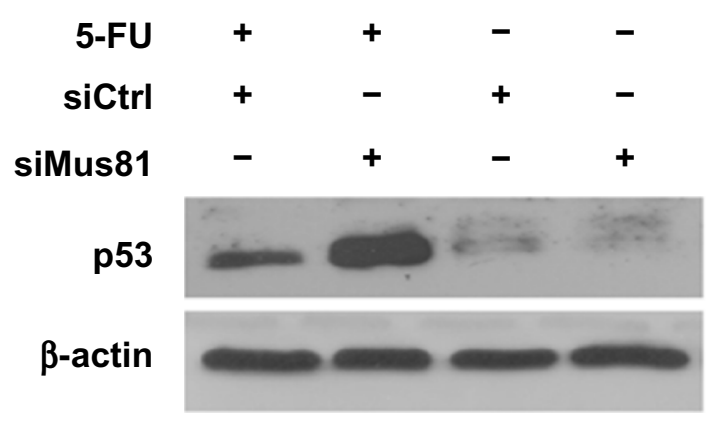

Figure 6 Western blot analysis of p53 expression in MCF-7 cells. Note: The expression of $p 53$ was significantly upregulated in the siMus 81 group after 5-FU challenge.

Abbreviations: 5-FU, 5-fluorouracil; siCtrl, control siRNA; siMus8I, Mus8I siRNA; siRNA, small interfering RNA.

deficient in Mus 81 or Eme1 have previously been shown to be selectively hypersensitive to DNA-damaging agents that result in stalled replication forks..$^{9,11,19-21}$ It has also been reported that Mus81 was a marker of cisplatin resistance in different kinds of tumor cells. ${ }^{13}$

In this study, we inhibited Mus81 expression by siMus 81 and examined the changes of chemotherapy sensitivity of MCF-7 and T47D cells to 5-FU. The results of quantitative RT-PCR and Western blot demonstrated that the mRNA and protein expression of Mus81 could be significantly suppressed by all three of the siMus $81 \mathrm{~s}$, especially siMus $81-3$. Thus, we chose siMus81-3 for the proceeding studies. Cell viability assay and colony formation assay are two classic experiments for examining the sensitivity of cancer cells to drugs. Cell viability is a common effect of proliferation and apoptosis. Clone formation rate reflects the cell group dependence and single cell proliferation ability by DNA-damaging stress. By using CCK-8 and plate colony formation assay, we found that both the cell viability and clonogenic survival of MCF-7 and T47D cells with 5-FU were significantly decreased after Mus81 inhibition. The above data reveal that Mus81 inhibition can improve the sensitivity of breast carcinoma cells to 5 -FU via reducing cell proliferation.

Cell cycle arrest has been demonstrated to be involved in the growth inhibition by DNA-damaging drugs. Our study revealed that Mus81 suppression by siRNA could affect the cell cycle distribution of MCF-7 and T47D cells by 5-FU treatment and that the proportion of G2 period was significantly increased in the siMus 81 group compared to in the siCtrl group. Moreover, another study has shown that Mus 81 silenced by gene targeting could activate the intra-S-phase and $\mathrm{G} 2 / \mathrm{M}$ checkpoints of cancer cells, thereby inducing an increase in sensitivity to MMC, MMS, cisplatin, ultraviolet radiation, ionizing radiation, and hydroxyurea. ${ }^{19}$ Pamidi et al also found that treatment with MMC resulted in an accumulation in the G2 phase of Mus81-deficient cells. ${ }^{22}$ These results are consistent with our study. Thus, cell cycle arrest might contribute to enhanced sensitivity by Mus 81 deficiency.

Annexin $\mathrm{V}$ analysis proved that the loss of Mus81 increased the apoptosis of MCF-7 cells treated with 5-FU. Mus81 inhibition can improve the sensitivity of MCF-7 cells to 5-FU via increasing cell apoptosis; however, Mus81 inhibition did not affect the apoptosis of T47D cells treated with 5-FU. We hypothesize that influence of Mus81 on apoptosis is different in different cells. The mechanism for this remains unclear.

p53 is one of the important cell cycle regulatory proteins and is able to induce cell apoptosis. DNA damage signal through ATM/ATR or via CHK1/CHK2 can activate p53 in a direct or indirect manner, respectively. ${ }^{23}$ p53 can upregulate the cell cycle inhibitory proteins and induce cell apoptosis. ${ }^{24}$ Mus $81^{-/-}$mouse embryonic fibroblasts display an elevated level of p53 in response to MMC. Furthermore, MMC sensitivity of Mus81 $81^{-/}$cells returned to wild-type level after inhibiting the activity of $\mathrm{p} 53 .{ }^{22}$ In our study, we found that the p53 expression level of MCF-7 cells in the siMus81 group increased more remarkably than that in the siCtrl group after 5-FU exposure. We speculated that Mus81 inhibition enhanced apoptosis of MCF-7 cells to 5-FU via upregulation of $\mathrm{p} 53$.

\section{Conclusion}

This study shows that Mus81 silenced by siRNA can suppress the cell viability and colony formation of MCF-7 and T47D cells. Hence, inhibition of Mus 81 by siRNA enhances sensitivity to 5-FU in breast carcinoma cells, mainly via reducing cell proliferation. We will further study the mechanism of Mus81 participating in chemosensitivity in the future. We will verify effectiveness of siMus81 in an animal model.

\section{Acknowledgments}

This study was supported by the Natural Science Foundation of Zhejiang Province (Y14H200002), Medicines Health Platform Key Project of Zhejiang Province (2013ZDA024), and Key Science and Technology Innovation Team of Zhejiang Province (2012R100-48-09).

\section{Disclosure}

The authors report no conflicts of interest in this work. 


\section{References}

1. Youlden DR, Cramb SM, Dunn NA, Muller JM, Pyke CM, Baade PD. The descriptive epidemiology of female breast cancer: an international comparison of screening, incidence, survival and mortality. Cancer Epidemiol. 2012;36(3):237-248.

2. Ferlay J, Shin HR, Bray F, Forman D, Mathers C, Parkin DM. Estimates of worldwide burden of cancer in 2008: GLOBOCAN 2008. Int $J$ Cancer. 2010;127(12):2893-2917.

3. Porkka K, Blomqvist C, Rissanen P, Elomaa I, Pyrhönen S. Salvage therapies in women who fail to respond to first-line treatment with fluorouracil, epirubicin, and cyclophosphamide for advanced breast cancer. J Clin Oncol. 1994;12(8):1639-1647.

4. He Z, Yuan J, Chen Z, Liu S, Shen Z, Fei H. [Chemosensitivity test for 170 human breast carcinoma samples]. Hunan Yi Ke Da Xue Xue Bao. 1998;23(6):531-534. Chinese.

5. Interthal H, Heyer WD. MUS81 encodes a novel helix-hairpin-helix protein involved in the response to UV- and methylation-induced DNA damage in Saccharomyces cerevisiae. Mol Gen Genet. 2000;263(5): 812-827.

6. Boddy MN, Lopez-Girona A, Shanahan P, Interthal H, Heyer WD, Russell P. Damage tolerance protein Mus81 associates with the FHA1 domain of checkpoint kinase Cds1. Mol Cell Biol. 2000;20(23): 8758-8766.

7. Chen XB, Melchionna R, Denis CM, et al. Human Mus81-associated endonuclease cleaves Holliday junctions in vitro. Mol Cell. 2001;8(5): 1117-1127.

8. Hartung F, Suer S, Bergmann T, Puchta H. The role of AtMUS81 in DNA repair and its genetic interaction with the helicase AtRecQ4A. Nucleic Acids Res. 2006;34(16):4438-4448.

9. Hanada K, Budzowska M, Davies SL, et al. The structure-specific endonuclease Mus81 contributes to replication restart by generating double-strand DNA breaks. Nat Struct Mol Biol. 2007;14(11): 1096-1104.

10. McPherson JP, Lemmers B, Chahwan R, et al. Involvement of mammalian Mus81 in genome integrity and tumor suppression. Science. 2004;304(5678):1822-1826.

11. Dendouga N, Gao H, Moechars D, Janicot M, Vialard J, McGowan CH. Disruption of murine Mus81 increases genomic instability and DNA damage sensitivity but does not promote tumorigenesis. Mol Cell Biol. 2005;25(17):7569-7579.
12. Tamblyn L, Li E, Sarras H, Srikanth P, Hande MP, McPherson JP. A role for Mus81 in the repair of chromium-induced DNA damage. Mutat Res. 2009;660(1-2):57-65.

13. Tomoda Y, Katsura M, Okajima M, Hosoya N, Kohno N, Miyagawa K. Functional evidence for Eme1 as a marker of cisplatin resistance. Int $J$ Cancer. 2009;124(12):2997-3001.

14. Feng XJ, Fu Z, Bai JF, Shi Y, Sun YM, Zhao HL. Identification of genes associated with 5-fluorouracial resistance in human pancreatic cancer cells. Acta Universitatis Medicinalis Nanjing (Natural Science). 2010;30(6):756-762.

15. Boddy MN, Gaillard PH, McDonald WH, Shanahan P, Yates JR 3rd, Russell P. Mus81-Eme1 are essential components of a Holliday junction resolvase. Cell. 2001;107(4):537-548.

16. Osman F, Whitby MC. Exploring the roles of Mus81-Eme1/Mms4 at perturbed replication forks. DNA Repair (Amst). 2007;6(7):1004-1017.

17. Murfuni I, Nicolai S, Baldari S, et al. The WRN and MUS81 proteins limit cell death and genome instability following oncogene activation. Oncogene. 2013;32(5):610-620.

18. Negrini S, Gorgoulis VG, Halazonetis TD. Genomic instability - an evolving hallmark of cancer. Nat Rev Mol Cell Biol. 2010;11(3):220-228.

19. Hiyama T, Katsura M, Yoshihara T, et al. Haploinsufficiency of the Mus81-Eme1 endonuclease activates the intra-S-phase and G2/M checkpoints and promotes rereplication in human cells. Nucleic Acids Res. 2006;34(3):880-892

20. Hanada K, Budzowska M, Modesti M, et al. The structure-specific endonuclease Mus81-Eme1 promotes conversion of interstrand DNA crosslinks into double-strands breaks. EMBO J. 2006;25(20):4921-4932.

21. Shimura T, Torres MJ, Martin MM, et al. Bloom's syndrome helicase and Mus81 are required to induce transient double-strand DNA breaks in response to DNA replication stress. J Mol Biol. 2008;375(4): $1152-1164$

22. Pamidi A, Cardoso R, Hakem A, et al. Functional interplay of p53 and Mus81 in DNA damage responses and cancer. Cancer Res. 2007;67(18): 8527-8535.

23. Ou YH, Chung PH, Sun TP, Shieh SY. p53 C-terminal phosphorylation by $\mathrm{CHK} 1$ and $\mathrm{CHK} 2$ participates in the regulation of DNA-damage-induced C-terminal acetylation. Mol Biol Cell. 2005;16(4):1684-1695.

24. Meek DW. The p53 response to DNA damage. DNA Repair (Amst). 2004;3(8-9):1049-1056.
OncoTargets and Therapy

\section{Publish your work in this journal}

OncoTargets and Therapy is an international, peer-reviewed, open access journal focusing on the pathological basis of all cancers, potential targets for therapy and treatment protocols employed to improve the management of cancer patients. The journal also focuses on the impact of management programs and new therapeutic agents and protocols on

\section{Dovepress}

patient perspectives such as quality of life, adherence and satisfaction The manuscript management system is completely online and includes a very quick and fair peer-review system, which is all easy to use. Visit http://www.dovepress.com/testimonials.php to read real quotes from published authors. 